\title{
A fluorescence probe study on the effects of surfactants on cloud points in aqueous poly( $N$-isopropylacrylamide) solutions
}

\author{
Masashi Osa, Yu Itoda, Yuki Suzuki, Takaya Yumoto and Ayaka Yoshida \\ Fluorescence probe methods were used to investigate micelle formation of poly( $N$-isopropylacrylamide) (PNIPA) with two types of \\ surfactants, anionic sodium $n$-dodecyl sulfate (SDS) and cationic $n$-dodecyltrimethylammonium chloride (DTAC), in aqueous \\ solutions using pyrene or 1-pyrenecarboxaldehyde as a fluorescence probe. Two PNIPA samples, one having a hydrophobic \\ chain-end group and the other having a negatively charged hydrophilic chain-end group, were used to investigate the effects of \\ the chain-end group on the formation of the micelles. The critical aggregation concentration, at which surfactant molecules \\ begin to bind to PNIPA chains to form micelles, was observed to be much lower for PNIPA solutions containing SDS than for \\ solutions containing DTAC. This is consistent with previous results showing that the cloud point in PNIPA solutions containing \\ SDS begins to increase from its value in surfactant-free solutions at a much lower concentration of added surfactant than that in \\ solutions containing DTAC. It was also observed that there is a discrepancy in the emission spectra of solutions containing SDS \\ between the two PNIPA samples but not for solutions containing DTAC, indicating that the chain-end groups of PNIPA may \\ affect the microenvironmental polarity in the micelles composed of PNIPA and the surfactants.
}

Polymer Journal (2015) 47, 59-65; doi:10.1038/pj.2014.93; published online 5 November 2014

\section{INTRODUCTION}

Recently, we performed a series of experimental studies on the phase behavior of aqueous poly( $N$-isopropylacrylamide) (PNIPA) solutions, ${ }^{1-5}$ which exhibited lower critical solution temperature miscibility near human body temperature that was caused by the breakdown of hydrogen bonds between polymers and the surrounding water molecules. During the course of these studies, ${ }^{1-5}$ we observed that the phase behavior was not as simple as it is generally considered to be and that cloud-point curves were affected considerably by the nature of the PNIPA chain-end groups, as well as by the degree of branching.

It is known that the phase behavior of aqueous PNIPA solutions is also influenced by the addition of surfactants. ${ }^{6}$ Until now, not a few studies have been performed regarding the effects of surfactants on the phase behavior of aqueous PNIPA solutions. ${ }^{7-15}$ In our previous work, ${ }^{16}$ we evaluated the effects of two types of surfactants, anionic sodium $n$-dodecyl sulfate (SDS) and cationic $n$-dodecyltrimethylammonium chloride (DTAC), on the cloud points in aqueous PNIPA solutions. In that study, ${ }^{16}$ we used two low-molecular-weight PNIPA samples, one having a hydrophobic chain-end group (M sample) and the other having a hydrophilic, negatively charged chain-end group ( $\mathrm{R}$ sample), to determine whether chain-end groups affect the cloud points. Figure 1 reproduces the plots in Osa et al. ${ }^{16}$ of the cloud points in aqueous PNIPA solutions (polymer mass concentration $c_{\mathrm{p}}=10 \mathrm{mg} \mathrm{ml}^{-1}$ ) with the addition of two surfactants, SDS or DTAC, against the concentration $\left(c_{\mathrm{s}}\right)$ of the added surfactant. The figure shows that the cloud points of both PNIPA samples with SDS increase monotonically and steeply with increasing $c_{s}$. By contrast, the cloud point for the M sample with DTAC is constant for $c_{\mathrm{s}} \lesssim 27 \mathrm{mM}$, and increases with increasing $c_{\mathrm{s}}$ for $c_{\mathrm{s}} \gtrsim 27 \mathrm{~mm}$. As for the solution of the R sample with DTAC, it was observed that the cloud point first decreases with increasing $c_{\mathrm{s}}$, then passes through a minimum at $c_{\mathrm{s}} \simeq 11 \mathrm{~mm}$, and finally increases for $c_{\mathrm{s}} \gtrsim 11 \mathrm{~mm}$. This indicates that the cloud point, as a function of $c_{\mathrm{s}}$, depends on the type of surfactant, as well as on the PNIPA chain-end group.

The above-mentioned increase in the cloud points in all the aqueous PNIPA solutions in the presence of surfactants is considered to result from electrostatic repulsion between charged micelles that are formed by binding of surfactant molecules to the PNIPA chains through hydrophobic interactions, as previously suggested by Schild and Tirrell. ${ }^{8}$ In the present study, we performed a detailed examination of the formation of PNIPA micelles with surfactant molecules using a fluorescence probe method. We used two types of fluorescence-probe molecules, pyrene and 1-pyrenecarboxaldehyde, to comprehensively examine micelle formation. As seen in Figure 1, the cloud point in the aqueous PNIPA solutions containing SDS begins to increase at a much lower $c_{\mathrm{S}}$ than that in the solutions containing DTAC, implying that SDS may bind to PNIPA to form micelles at a much lower $c_{\mathrm{s}}$ than DTAC. The original aim of this study was to confirm this expectation. However, as seen in Figure 1, 


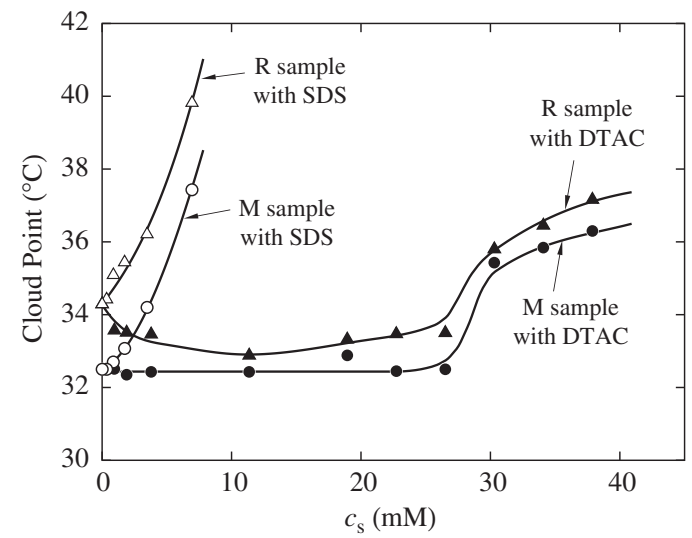

Figure 1 Plots of the cloud point in aqueous PNIPA solutions against the concentration $c_{\mathrm{S}}$ of the added surfactants: $(O)$, the $M$ sample with an addition of SDS; $(\bullet)$, the M sample with an addition of DTAC; $(\triangle)$, the R sample with an addition of SDS; $(\boldsymbol{\Lambda})$, the $\mathrm{R}$ sample with an addition of DTAC. ${ }^{16}$ The values of the weight-average molecular weight $M_{\mathrm{w}}$ are $6.45 \times 10^{4}$ and $3.26 \times 10^{4}$ for the $M$ and $R$ samples, respectively. In all the solutions, the concentration $c_{p}$ of the PNIPA samples is $10 \mathrm{mg} \mathrm{ml}^{-1}$. The solid curves connect smoothly the respective data points.

the decrease in the cloud point observed for the solution of the $\mathrm{R}$ sample containing DTAC at low $c_{\mathrm{s}}$, is presumed to arise because of the cationic DTAC molecules bound to the negatively charged chain ends of the R sample, which diminishes the hydrophilicity of its chain end. The investigation of the effects of the chain-end groups on the binding of surfactant molecules to PNIPA chains was the second goal of this study.

\section{EXPERIMENTAL PROCEDURE}

\section{Materials}

The PNIPA sample M5 used in this study was prepared by fractional precipitation of the original sample, which had been previously synthesized by radical polymerization in methanol using azobisisobutyronitrile as an initiator. ${ }^{1}$ After fractionation, the sample was purified and dried as described in our previous paper. ${ }^{1}$ We note that almost all of the initiating and terminating chain ends of the sample M5 are considered to be hydrophobic isobutyronitrile groups derived from azobisisobutyronitrile.

The PNIPA sample R5 was prepared by aqueous redox polymerization using a redox catalyst consisting of ammonium persulfate, $\left(\mathrm{NH}_{4}\right)_{2} \mathrm{~S}_{2} \mathrm{O}_{8}$ (Nacalai Tesque, Inc., Kyoto, Japan), as the oxidant and sodium metabisulfite, $\mathrm{Na}_{2} \mathrm{~S}_{2} \mathrm{O}_{5}$ (Nacalai Tesque Inc.), as the reductant, according to the procedure reported by Wooten et al. ${ }^{17}$ The procedure used for the polymerization and purification of the sample was the same as that described in our previous paper, ${ }^{5}$ except the present sample was not fractionated. We note that almost all of the initiating chain ends of the sample R5 are considered to be anionic sulfonate groups. ${ }^{5}$ As for the terminating chain-end groups of the sample R5, we do not have any detailed information. However, at least, it may be considered that the terminating chain-end groups of the sample is not positively charged.

The weight-average molecular weight, $M_{\mathrm{w}}$, and the ratio of the $M_{\mathrm{w}}$ to the number-average molecular weight, $M_{\mathrm{n}}$, for the samples M5 and R5 were determined using analytical gel permeation chromatography with two serially connected columns, SB-805HQ and SB-804HQ (Showa Denko KK, Tokyo, Japan), connected to a L-7100 solvent delivery pump (Hitachi, Ltd, Tokyo, Japan) and a RI-930 refractive index detector (JASCO Corporation). $\mathrm{N}, \mathrm{N}$-dimethylformamide containing $10 \mathrm{~mm}$ lithium bromide at $50^{\circ} \mathrm{C}$ was used as an eluent, and 12 standard polystyrene samples (Tosoh Corporation, Tokyo, Japan, $M_{\mathrm{w}}=550-5.46 \times 10^{6}$ ) were used as reference standards. The values of $M_{\mathrm{W}}$ and $M_{\mathrm{w}} / M_{\mathrm{n}}$ that were determined for the two samples are listed in Table 1 . We note that both of the samples are atactic. ${ }^{1,5} \mathrm{We}$ also note that both samples are considered to have a small number of branch points. ${ }^{1,5}$
Table 1 Values of $M_{\mathrm{w}}$ and $M_{\mathrm{w}} / M_{\mathrm{n}}$ for poly $(N$-isopropylacrylamide)

\begin{tabular}{lcc}
\hline Sample & $\mathrm{M}_{w}$ & $\mathrm{M}_{w} \mathrm{M}_{n}$ \\
\hline M5 & $5.16 \times 10^{4}$ & 1.10 \\
R5 & $5.80 \times 10^{4}$ & 1.51 \\
\hline
\end{tabular}

The surfactants SDS (Wako Pure Chemical Industries, Ltd, Osaka, Japan) and DTAC (Tokyo Chemical Industry Co., Ltd., Tokyo, Japan) that were added to the aqueous PNIPA solutions in the fluorescence measurements were of reagent grade. Pyrene (Nacalai Tesque Inc., Kyoto, Japan) and 1-pyrenecarboxaldehyde (Tokyo Chemical Industry Co., Ltd.) that were used as fluorescence probes were of reagent grade. Water used for the fluorescence measurements was highly purified through an Autopure WT101UV water purification system (Yamato Scientific Co., Ltd, Tokyo, Japan), and its resistivity was $18.2 \mathrm{M} \Omega \mathrm{cm}$.

\section{Fluorescence measurements}

For the aqueous solutions of the PNIPA samples M5 and R5 with the addition of SDS or DTAC in which a small amount of pyrene or 1-pyrenecarboxaldehyde was solubilized as the fluorescence probe, steadystate fluorescence spectra were obtained using a JASCO FP-6300 fluorescence spectrometer equipped with an EHC-573 peltier thermostatted single cell holder (JASCO Corporation, Tokyo, Japan). The solutions used for the fluorescence measurements were prepared according to a standard procedure reported in literatures ${ }^{8,18}$ as follows: Aliquots $(10 \mu \mathrm{l})$ of an acetone solution of pyrene or 1-pyrenecarboxaldehyde $\left(1 \times 10^{-3} \mathrm{M}\right)$ were transferred to sample vials and then the acetone was removed by evaporation. Subsequently, $10 \mathrm{ml}$ of aqueous PNIPA solutions containing SDS or DTAC was added to the vials with vigorous stirring to obtain a final pyrene or 1-pyrenecarboxaldehyde concentration of $\sim 1 \times 10^{-6} \mathrm{M}$. The PNIPA concentration $\left(c_{\mathrm{p}}\right)$ was chosen to be $1.0 \mathrm{mg} \mathrm{ml}^{-1}$ in all of the measurements, and the surfactant concentration was varied between 0 and $52 \mathrm{~mm}$ for SDS, and between 0 and $53 \mathrm{~mm}$ for DTAC. Each solution was rendered homogeneous by continuous stirring at room temperature for 1 day.

Emission spectra of pyrene and 1-pyrenecarboxaldehyde in the aqueous solutions were measured using an excitation wavelength of 337 and $365.5 \mathrm{~nm}$, respectively, at $25.0^{\circ} \mathrm{C}$. Slit widths were maintained at $2.5 \mathrm{~nm}$ for pyrene and at $5 \mathrm{~nm}$ for 1-pyrenecarboxaldehyde during the measurements. For the solutions solubilizing pyrene, the ratio $I_{1} / I_{3}$ of the fluorescence intensities of the 0,0 band $(373 \mathrm{~nm})$ and the 0,2 band $(383 \mathrm{~nm})$ in the emission spectra was determined as a function of the surfactant concentration. For the solutions solubilizing 1-pyrenecarboxaldehyde, the emission wavelength maximum, $\lambda_{\max }$, in the emission spectra was determined as a function of the surfactant concentration. In addition, to examine the temperature dependence of $I_{1} / I_{3}$ (of pyrene) and $\lambda_{\max }$ (of 1-pyrenecarboxaldehyde), the measurements were also performed on the following aqueous solutions of the samples M5 and R5 at $c_{\mathrm{p}}=1.0 \mathrm{mg} \mathrm{ml}^{-1}$ : the solutions without surfactant at various temperatures ranging from 26 to $37^{\circ}$ C, those with $0.35 \mathrm{~mm}$ SDS at various temperatures ranging from 26 to $38^{\circ} \mathrm{C}$, those with $3.5 \mathrm{~mm}$ SDS at various temperatures ranging from 26 to $60^{\circ} \mathrm{C}$, those with $11 \mathrm{~mm}$ DTAC at various temperatures ranging from 26 to $36^{\circ} \mathrm{C}$, and those with $23 \mathrm{~mm}$ DTAC at various temperatures ranging from 26 to $39^{\circ} \mathrm{C}$.

\section{RESULTS AND DISCUSSION}

\section{Emission spectra}

Figure 2 shows examples of emission spectra of pyrene and 1-pyrenecarboxaldehyde solubilized in aqueous solutions of the sample M5 with additions of 0.35 and $3.5 \mathrm{~mm}$ SDS at $25.0^{\circ} \mathrm{C}$, each at $c_{\mathrm{p}}=1.0 \mathrm{mg} \mathrm{ml}^{-1}$. The solid and dashed curves represent the fluorescence intensities for pyrene and 1-pyrenecarboxaldehyde, respectively, at the indicated concentrations of SDS. The ratio $I_{1} / I_{3}$ of the first $(373 \mathrm{~nm})$ peak intensity to the third $(383 \mathrm{~nm})$ of pyrene is 1.73 for the solution with $0.35 \mathrm{~mm}$ SDS, whereas it is 1.07 for the solution with $3.5 \mathrm{~mm}$ SDS. As is well known, the ratio $I_{1} / I_{3}$ reflects the 


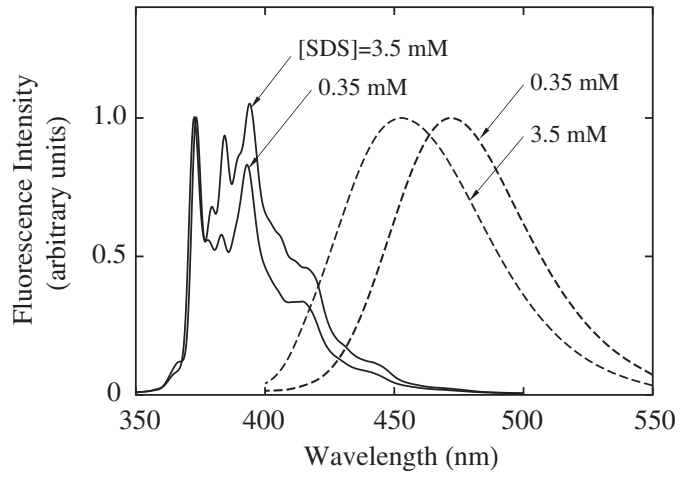

Figure 2 Emission spectra of pyrene and 1-pyrenecarboxaldehyde solubilized in aqueous solutions of the sample M5 with additions of $0.35 \mathrm{~mm}$ SDS and $3.5 \mathrm{~mm}$ SDS at $25.0^{\circ} \mathrm{C}$ and at $c_{\mathrm{p}}=1.0 \mathrm{mg} \mathrm{ml}^{-1}$. The solid and dashed curves represent the values of fluorescence intensities for pyrene and 1-pyrenecarboxaldehyde, respectively, at the indicated values of the concentration of SDS.

microenvironmental polarity surrounding the fluorescence probe pyrene, which has a high value when pyrene is in polar media, but decreases as pyrene is solubilized in a more hydrophobic (or nonpolar) environment of micelles that form in the solution. ${ }^{8,19,20}$ The value of 1.73 that was obtained for $I_{1} / I_{3}$ is similar to the value of 1.74 that was obtained for the aqueous solution of the sample M5 without surfactant at the same $c_{\mathrm{p}}$ and at the same temperature, indicating that practically no micelles are present in the solution with $0.35 \mathrm{~mm}$ SDS, and the pyrene molecules are considered to be dispersed in the aqueous solution. The latter value of 1.07 for $I_{1} / I_{3}$ indicates that micelles are formed in the solution with $3.5 \mathrm{~mm}$ SDS, and that most of the pyrene molecules in the solution are incorporated into the micelles.

By contrast, 1-pyrenecarboxaldehyde exhibits a blue shift in the emission spectra as the microenvironmental polarity surrounding the probe molecule is decreased, and this can be used as a fluorescence probe to investigate the formation of micelles. ${ }^{8,21}$ As shown in Figure 2, the $\lambda_{\max }$ values for 1-pyrenecarboxaldehyde are 472 and $453 \mathrm{~nm}$ for the aqueous solutions of the sample M5 with 0.35 and $3.5 \mathrm{~mm}$ SDS, respectively. We note that the $\lambda_{\max }$ for the aqueous solution of the sample M5 $\left(c_{\mathrm{p}}=1.0 \mathrm{mg} \mathrm{ml}^{-1}\right)$ without surfactant at $25.0^{\circ} \mathrm{C}$ is $472 \mathrm{~nm}$, which is equal to the value obtained for the aqueous PNIPA solution with $0.35 \mathrm{~mm}$ SDS. As the SDS concentration is increased to $3.5 \mathrm{mM}$, the $\lambda_{\max }$ shifts to a lower wavelength of $453 \mathrm{~nm}$. These results are consistent with those obtained for the solutions solubilizing pyrene, that is, micelles are not formed in the solution with $0.35 \mathrm{~mm}$ SDS, whereas a large number of micelles are formed in the solution with $3.5 \mathrm{~mm}$ SDS.

\section{Surfactant concentration dependence}

Aqueous PNIPA solutions containing SDS. In this section, we examine the behavior of the $I_{1} / I_{3}$ ratio of pyrene and the $\lambda_{\max }$ of 1-pyrenecarboxaldehyde in aqueous PNIPA solutions, which are determined in the same manner as those described in the previous section, as a function of the concentration of added surfactant. Figure 3a shows plots of $I_{1} / I_{3}$ of pyrene against the SDS concentration ([SDS]) for aqueous solutions containing SDS at $25.0^{\circ} \mathrm{C}$. The unfilled circles represent the values for the aqueous solutions of the sample M5, and the unfilled triangles represent those for the aqueous solutions of the sample $\mathrm{R} 5$, both at $c_{\mathrm{p}}=1.0 \mathrm{mg} \mathrm{ml}^{-1}$. The filled diamonds represent the values for aqueous SDS solutions without
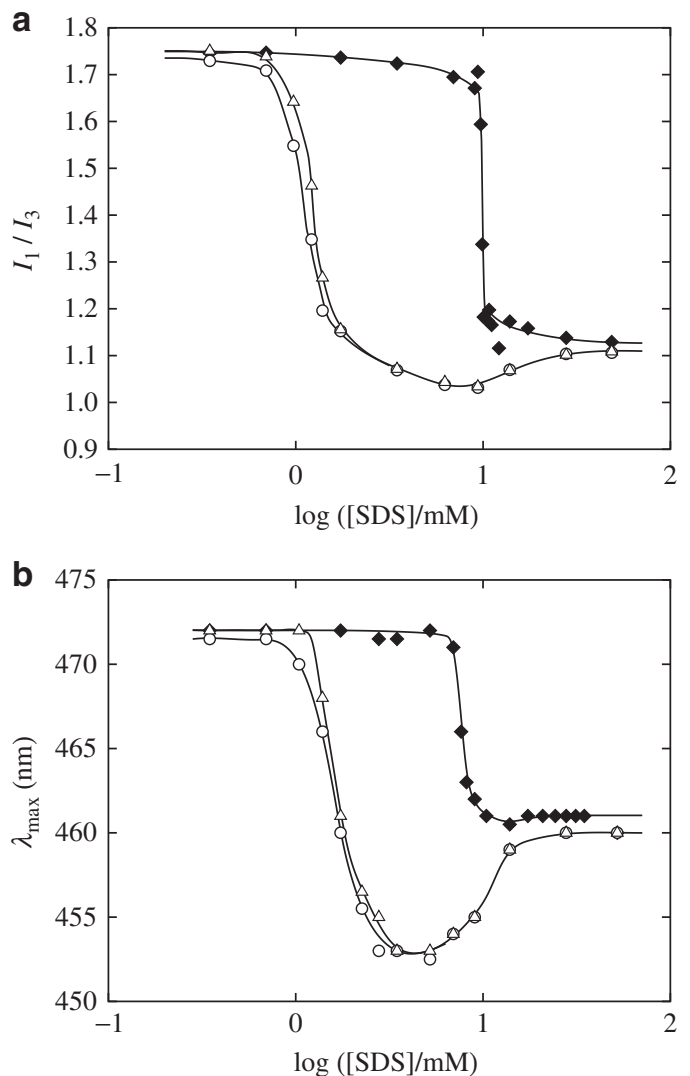

Figure 3 Plots of $l_{1} / l_{3}$ of pyrene (a) or $\lambda_{\max }$ of 1 -pyrenecarboxaldehyde (b) in aqueous solutions of the sample M5 $(O)$ and in those of the sample R5 $(\triangle)$, both containing SDS, and in aqueous SDS solutions without PNIPA ( $)$ against the concentration of SDS at $25^{\circ} \mathrm{C}$. In the PNIPA solutions, $c_{p}$ is $1.0 \mathrm{mg} \mathrm{ml}^{-1}$. The solid curves connect smoothly the respective data points.

PNIPA. It can be seen from the figure that $I_{1} / I_{3}$ for the aqueous solutions without PNIPA decreases gradually with increasing [SDS] and decreases abruptly from $\sim 1.7$ to $\sim 1.1$ at $[\mathrm{SDS}] \simeq 9 \mathrm{~mm}$. This threshold value of [SDS] is regarded as the critical micelle concentration (CMC) of SDS, at which the SDS molecules begin to form (homo)micelles. This value of the CMC for SDS is in good agreement with literature values. ${ }^{8,22}$ It can also be seen from the figure that $I_{1} / I_{3}$ for the aqueous solutions of the PNIPA sample, either M5 or R5, containing SDS decreases abruptly from $\sim 1.7$ at a much lower [SDS] $(\simeq 0.9 \mathrm{~mm}$ ) than that for the PNIPA-free solution. This result is considered to reflect the fact that the SDS molecules begin to bind to the PNIPA chains to form complex micelles that are composed of PNIPA and SDS well below the CMC for SDS. The surfactant concentration at which the surfactant molecules and polymer chains begin to form such complex micelles is called the critical aggregation concentration (CAC). The CAC for SDS in PNIPA solutions is estimated to be $\sim 0.9 \mathrm{~mm}$, which is comparable to values reported in the literature. ${ }^{8,10}$

Figure $3 \mathrm{~b}$ shows plots of the $\lambda_{\max }$ of 1-pyrenecarboxaldehyde against [SDS] in aqueous solutions containing SDS at $25.0^{\circ} \mathrm{C}$. The symbols have the same meaning as those in Figure $3 \mathrm{a}$. As in the case of $I_{1} / I_{3}$ shown in Figure $3 \mathrm{a}$, the $\lambda_{\max }$ for aqueous PNIPA solutions begins to decrease at an SDS concentration $\sim 10$ times lower than that for the PNIPA-free solution, confirming the above conclusion that the CAC for SDS in PNIPA solutions is much lower than the CMC for SDS. 
Aqueous PNIPA solutions containing DTAC. Figure 4a shows plots of $I_{1} / I_{3}$ of pyrene against the DTAC concentration ([DTAC]) for aqueous solutions containing DTAC at $25.0^{\circ} \mathrm{C}$. The unfilled circles represent the values for the aqueous solutions of the sample M5, and the unfilled triangles represent those for the aqueous solutions of the sample R5, both at $c_{\mathrm{p}}=1.0 \mathrm{mg} \mathrm{ml}^{-1}$. The filled diamonds represent the values for the aqueous DTAC solutions without PNIPA. As seen in the figure, $I_{1} / I_{3}$ for aqueous DTAC solutions without PNIPA decreases abruptly from $\sim 1.7$ to $\sim 1.4$ at $[D T A C] \simeq 20 \mathrm{~mm}$. This value of [DTAC] is considered to be the CMC of DTAC, and it is in good agreement with the literature value. ${ }^{22} I_{1} / I_{3}$ for the aqueous solutions of the PNIPA sample, either M5 or R5, containing DTAC decreases abruptly from $\sim 1.7$ at $[\mathrm{DTAC}] \simeq 15 \mathrm{~mm}$, which is slightly lower than the CMC for DTAC estimated above, and is regarded as the CAC for DTAC in PNIPA solutions.

Figure $4 \mathrm{~b}$ shows plots of the $\lambda_{\max }$ of 1-pyrenecarboxaldehyde against [DTAC] for aqueous solutions containing DTAC at $25.0^{\circ} \mathrm{C}$. All the symbols have the same meaning as those in Figure 4a. The difference in the behavior of the $\lambda_{\max }$ between the aqueous PNIPA solutions and the solution without PNIPA is similar to the behavior of $I_{1} / I_{3}$ shown in Figure 4a.

As seen in Figure 1, the cloud point for the aqueous PNIPA solutions containing SDS begins to increase from the value for surfactant-free solutions at a much lower $c_{\mathrm{s}}$ than for the aqueous PNIPA solutions containing DTAC. In both the cases, the increase in the cloud point is thought to arise from the electrostatic repulsion
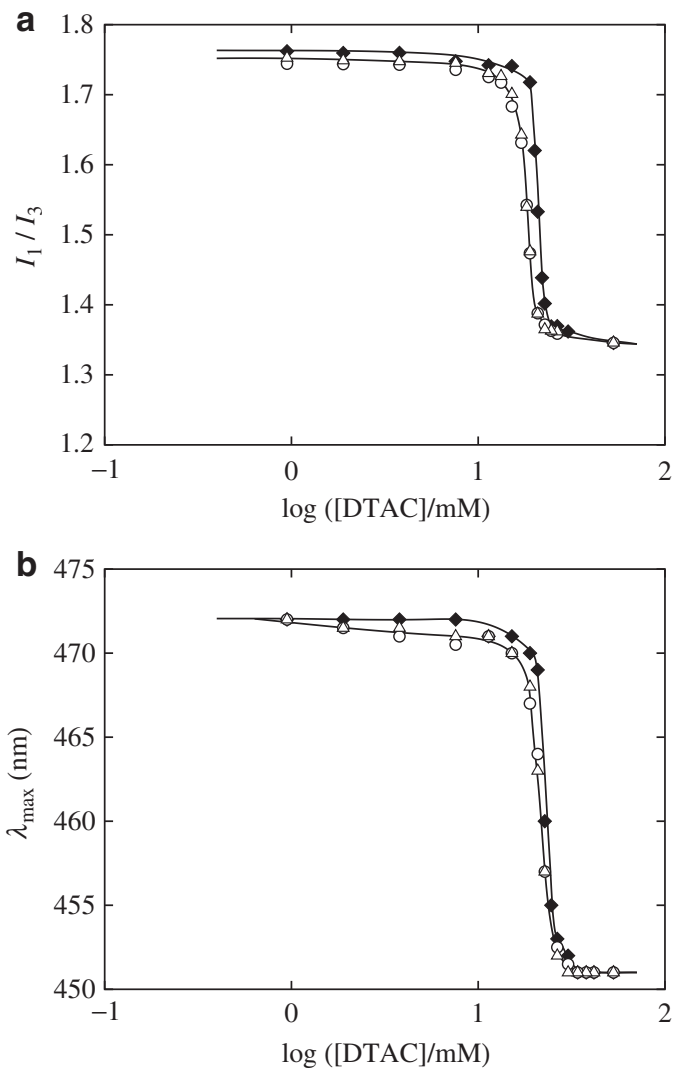

Figure 4 Plots of $I_{1} / l_{3}$ of pyrene (a) or $\lambda_{\max }$ of 1 -pyrenecarboxaldehyde (b) in aqueous solutions of the sample M5 $(O)$ and in those of the sample R5 $(\triangle)$, both containing DTAC, and in aqueous DTAC solutions without PNIPA ( ) against the concentration of DTAC at $25^{\circ} \mathrm{C}$. In the PNIPA solutions, $c_{p}$ is $1.0 \mathrm{mg} \mathrm{ml}^{-1}$. The solid curves connect smoothly the respective data points. between the charged micelles formed by binding of the surfactant molecules to the PNIPA chains. This electrostatic repulsion suppresses aggregation of the PNIPA chains, leading to an elevation of the cloud point. Because the CAC with SDS is much lower than the CAC with DTAC, it may be considered that the negatively charged micelles composed of PNIPA and SDS are formed at a much lower $c_{\mathrm{s}}$ than the positively charged micelles composed of PNIPA and DTAC. It may therefore be concluded that the difference in the value of the $c_{\mathrm{s}}$ at which the cloud point in the aqueous PNIPA solutions with SDS or DTAC begins to increase is caused by the difference in the CAC between the two surfactants.

\section{Temperature dependence}

Aqueous PNIPA solutions without surfactant. Next, we examined the temperature dependence of the $I_{1} / I_{3}$ ratio of pyrene and the $\lambda_{\max }$ of 1-pyrenecarboxaldehyde solubilized in aqueous PNIPA solutions with and without surfactant. Figures $5 \mathrm{a}$ and $\mathrm{b}$ show plots of $I_{1} / I_{3}$ and $\lambda_{\max }$, respectively, against temperature for the aqueous PNIPA solutions without surfactant. The unfilled circles represent the values for the aqueous solutions of the sample M5, and the unfilled triangles represent those for the aqueous solutions of the sample R5, both at $c_{\mathrm{p}}=1.0 \mathrm{mg} \mathrm{ml}^{-1}$. It can be seen that $I_{1} / I_{3}$ for the aqueous solutions of the two PNIPA samples decreases gradually with increasing temperature, and then decreases steeply at threshold temperatures, which are estimated to be $\sim 33.5^{\circ} \mathrm{C}$ and $\sim 35.0^{\circ} \mathrm{C}$ for the samples M5 and $\mathrm{R} 5$, respectively. The $\lambda_{\max }$ remains constant at lower temperatures, then decreases steeply at the same temperatures as observed with $I_{1} / I_{3}$. The sharp decrease in $I_{1} / I_{3}$ and $\lambda_{\max }$ is considered to reflect the fact that the PNIPA chains begin to form aggregates at the threshold temperature, and, subsequently, the fluorescence-probe molecules, which are dispersed in water at a lower temperature, are incorporated into the aggregates above the threshold temperature. Thus, the temperature at which $I_{1} / I_{3}$ or $\lambda_{\max }$ exhibits a rapid decrease may be regarded as being approximately equivalent to the cloud point in the aqueous PNIPA solutions. As pointed out in the previous paper, ${ }^{5}$ the cloud point in the aqueous solution of the $\mathrm{R}$ sample, which was determined from measurements of the transmittance of light passing through the solution at a given $c_{\mathrm{p}}$, is $\sim 1.5^{\circ} \mathrm{C}$ higher than that of the $\mathrm{M}$ sample at the same $c_{\mathrm{p}}$, if the values of the $M_{\mathrm{W}} \mathrm{s}$ for the two PNIPA samples are similar. This may be because the hydrophilicity of the chain-end group is stronger for the $\mathrm{R}$ sample than for the $\mathrm{M}$ sample. The same tendency was observed with the 'cloud point' determined above as the temperature at which the steep decrease in $I_{1} / I_{3}$ or $\lambda_{\max }$ occurs. In addition, Figure 5 shows that the values for $I_{1} / I_{3}$ or $\lambda_{\max }$ are slightly lower for the sample M5 than for the sample R5 over the entire range of temperatures examined, indicating that the microenvironment surrounding the fluorescence-probe molecules is more hydrophobic in the solution of the former sample than in the solution of the latter. Consequently, it may be concluded that the chain-end groups of the PNIPA chains may affect the microenvironmental polarity surrounding the probe molecules in the aqueous PNIPA solutions without surfactant.

Aqueous PNIPA solutions containing SDS. Figure 6a shows plots of $I_{1} / I_{3}$ of pyrene against temperature for aqueous PNIPA solutions containing SDS. The unfilled circles with pip up represent the values for the aqueous solution of the sample M5, and the unfilled triangles with pip up represent those for the solution of the sample R5, both at [SDS] $=0.35 \mathrm{~mm}$. The unfilled circles with pip down represent the values for the aqueous solution of the sample M5, and the unfilled triangles with pip down represent those for the solution of the sample 

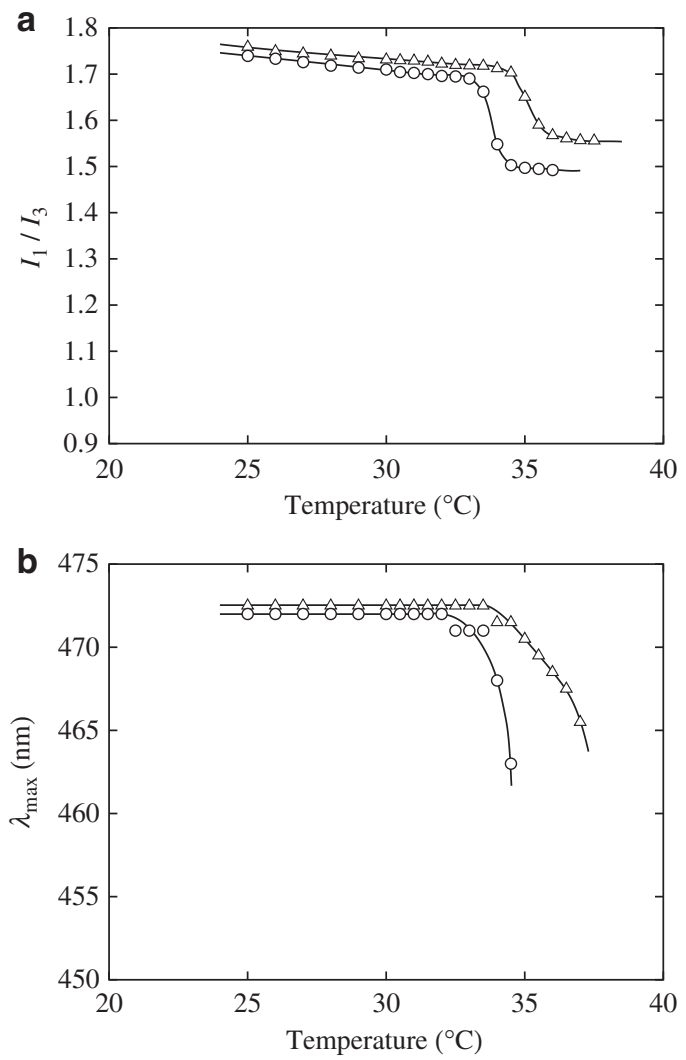

Figure 5 Plots of $I_{1} / l_{3}$ of pyrene (a) or $\lambda_{\max }$ of 1-pyrenecarboxaldehyde (b) in aqueous PNIPA solutions without surfactant against temperature: $(O)$, the sample M5; $(\triangle)$, the sample R5. In both the solutions, $c_{\mathrm{p}}$ is $1.0 \mathrm{mg} \mathrm{ml}^{-1}$. The solid curves connect smoothly the respective data points.

$\mathrm{R} 5$, both at $[\mathrm{SDS}]=3.5 \mathrm{~mm}$. In all of the solutions, the $c_{\mathrm{p}}$ is $1.0 \mathrm{mg} \mathrm{ml}^{-1}$. We note that SDS concentrations of 0.35 and $3.5 \mathrm{~mm}$ correspond to the values below and above the CAC for SDS, respectively, which was estimated at $25.0^{\circ} \mathrm{C}$ in the previous section. It can be seen that $I_{1} / I_{3}$ for aqueous solutions of the two PNIPA samples with $0.35 \mathrm{~mm}$ SDS exhibits similar behavior as that for the aqueous PNIPA solutions without surfactant shown in Figure 5a, that is, $I_{1} / I_{3}$ at $[\mathrm{SDS}]=0.35 \mathrm{~mm}$ decreases gradually with increasing temperature and then decreases rapidly at $\sim 33.5^{\circ} \mathrm{C}$ and $\sim 35.0^{\circ} \mathrm{C}$ for the samples M5 and R5, respectively. Furthermore, the $I_{1} / I_{3}$ for the aqueous solutions of the two PNIPA samples with $3.5 \mathrm{~mm}$ SDS decreased gradually with increasing temperature and then increased inversely at threshold temperatures, which are $\sim 49^{\circ} \mathrm{C}$ and $\sim 51^{\circ} \mathrm{C}$ for the samples M5 and R5, respectively. Because a SDS concentration of $3.5 \mathrm{~mm}$ is greater than the CAC for SDS, it is possible that SDS molecules form complex micelles with the PNIPA chains, and the pyrene molecules become incorporated in the hydrophobic microenvironment of the micelles in the solutions. The increase in the $I_{1} / I_{3}$ at $[\mathrm{SDS}]=3.5 \mathrm{mM}$, which begins at $\sim 49^{\circ} \mathrm{C}$ and $\sim 51^{\circ} \mathrm{C}$ for the samples M5 and R5, respectively, suggests that pyrene molecules are transferred to the more hydrophilic microenvironment as the temperature increases. Hence, we may speculate that the complex micelles that form at lower temperatures begin to collapse at the threshold temperature owing to shrinking and/or aggregation of the PNIPA chains, and the pyrene molecules are then excluded from the interior of the complex micelles, leading to the increase in $I_{1} / I_{3}$. Furthermore, it is important to note that there is a discrepancy in the $I_{1} / I_{3}$ values
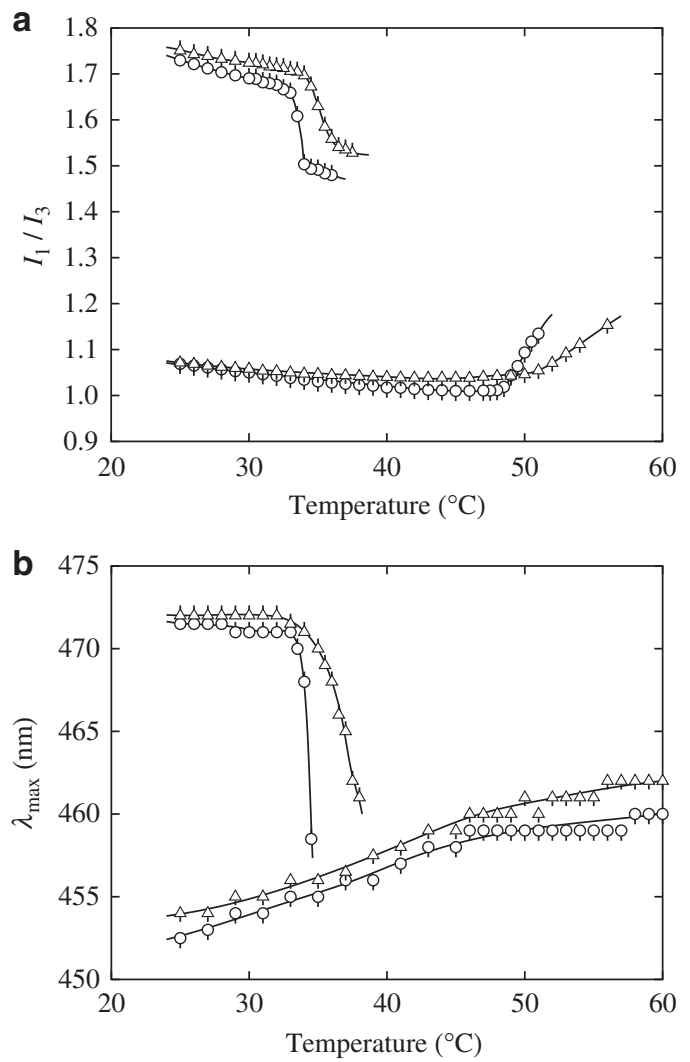

Figure 6 Plots of $l_{1} / l_{3}$ of pyrene (a) or $\lambda_{\max }$ of 1 -pyrenecarboxaldehyde (b) in aqueous PNIPA solutions with an addition of SDS against temperature: ( $\bigcirc$ with pip up), the sample M5 at $[S D S]=0.35 \mathrm{~mm}$; ( $\triangle$ with pip up), the sample $\mathrm{R} 5$ at $[S D S]=0.35 \mathrm{~mm}$; ( $\bigcirc$ with pip down), the sample M5 at $[S D S]=3.5 \mathrm{~mm}$; ( $\triangle$ with pip down), the sample $\mathrm{R} 5$ at $[S D S]=3.5 \mathrm{~mm}$. In all the solutions, $c_{p}$ is $1.0 \mathrm{mg} \mathrm{ml}^{-1}$. The solid curves connect smoothly the respective data points.

between the two PNIPA samples M5 and R5 at either value of [SDS]. This indicates that the chain-end groups of PNIPA may affect the microenvironmental polarity surrounding the pyrene molecules not only when the probe molecules are solubilized in the micelle-free PNIPA solutions containing SDS, but also when they are incorporated into PNIPA-SDS complex micelles.

Figure $6 \mathrm{~b}$ shows plots of the $\lambda_{\max }$ of 1-pyrenecarboxaldehyde against temperature for aqueous PNIPA solutions containing SDS. The symbols have the same meaning as those in Figure 6a. The behavior of the $\lambda_{\max }$ at $[\mathrm{SDS}]=0.35 \mathrm{~mm}$ for the aqueous solutions of the two PNIPA samples is similar to that of the $I_{1} / I_{3}$ at the same value of [SDS] shown in Figure $6 \mathrm{a}$, that is, the $\lambda_{\max }$ decreases steeply at $\sim 33.5^{\circ} \mathrm{C}$ and $\sim 35.0^{\circ} \mathrm{C}$ for the samples M5 and R5, respectively. However, the $\lambda_{\max }$ at [SDS] $=3.5 \mathrm{~mm}$ continues to increase gradually for both PNIPA samples with increasing temperature over the entire range of temperatures examined, in contrast to the case of $I_{1} / I_{3}$ at the same [SDS]. The difference in the behavior between $I_{1} / I_{3}$ and $\lambda_{\max }$ at $[\mathrm{SDS}]=3.5 \mathrm{~mm}$ may possibly be regarded as arising from the difference in the nature of the interactions between the fluorescenceprobe molecules and the complex micelles between the two fluorescence probes, because pyrene is a highly hydrophobic molecule, whereas 1-pyrenecarboxaldehyde is a relatively polar molecule because of its formyl group. At either value of [SDS], there is a discrepancy in the value of the $\lambda_{\max }$ between the two samples M5 and R5, confirming 

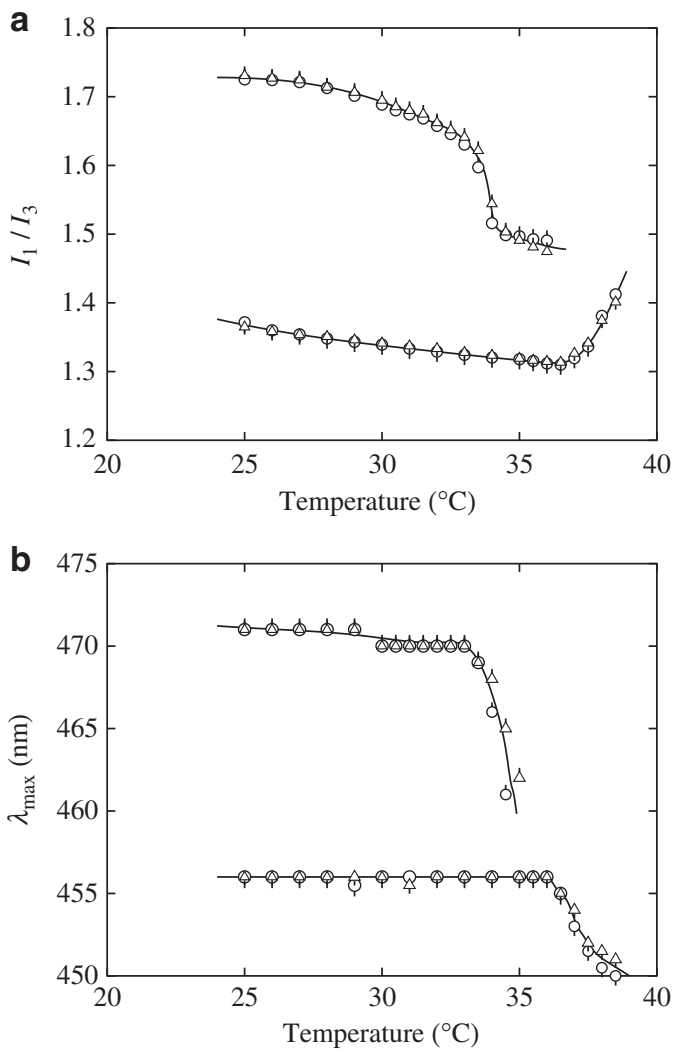

Figure 7 Plots of $l_{1} / l_{3}$ of pyrene (a) or $\lambda_{\max }$ of 1 -pyrenecarboxaldehyde (b) in aqueous PNIPA solutions with an addition of DTAC against temperature: ( $\bigcirc$ with pip up), the sample M5 at $[D T A C]=11 \mathrm{~mm}$; ( $\triangle$ with pip up), the sample $\mathrm{R} 5$ at $[D T A C]=11 \mathrm{~mm}$; ( $\bigcirc$ with pip down), the sample M5 at $[D T A C]=23 \mathrm{~mm}$; ( $\triangle$ with pip down), the sample R5 at $[D T A C]=23 \mathrm{~mm}$. In all the solutions, $c_{\mathrm{p}}$ is $1.0 \mathrm{mg} \mathrm{ml}^{-1}$. The solid curves connect smoothly the respective data points.

the above finding that the chain-end groups of the PNIPA chain may affect the microenvironmental polarity surrounding the fluorescence-probe molecules, not only in the micelle-free PNIPA solutions containing SDS but also in the complex micelles.

Aqueous PNIPA solutions containing DTAC. Figures $7 \mathrm{a}$ and $\mathrm{b}$ show plots of $I_{1} / I_{3}$ of pyrene and the $\lambda_{\max }$ of 1-pyrenecarboxaldehyde, respectively, against temperature for aqueous PNIPA solutions containing DTAC. The unfilled circles with pip up represent the values for the aqueous solution of the sample M5, and the unfilled triangles with pip up represent those for the solution of the sample R5, both at $[D T A C]=11 \mathrm{~mm}$. The unfilled circles with pip down represent the values for the aqueous solution of the sample M5, and the unfilled triangles with pip down represent those for the solution of the sample $\mathrm{R} 5$, both at $[\mathrm{DTAC}]=23 \mathrm{~mm}$. In all of the solutions, the $c_{\mathrm{p}}$ is $1.0 \mathrm{mg} \mathrm{ml}^{-1}$. We note that DTAC concentrations of 11 and $23 \mathrm{~mm}$ correspond to the values below and above the CAC for DTAC, respectively, which was estimated at $25.0^{\circ} \mathrm{C}$ in the previous section. Therefore, complex micelles between DTAC and PNIPA may be formed at $[\mathrm{DTAC}]=23 \mathrm{~mm}$ but not at $[\mathrm{DTAC}]=11 \mathrm{~mm}$. It can be seen that $I_{1} / I_{3}$ for the aqueous PNIPA solutions with $11 \mathrm{~mm}$ DTAC decreases gradually with increasing temperature and then decreases steeply at $\sim 34^{\circ} \mathrm{C}$, whereas that for the PNIPA solutions with $23 \mathrm{~mm}$ DTAC increases steeply at $\sim 37^{\circ} \mathrm{C}$. As for the $\lambda_{\max }$, it can be seen that the value for the aqueous PNIPA solutions with $11 \mathrm{~mm}$ DTAC decreases steeply at $\sim 34^{\circ} \mathrm{C}$, whereas that for the PNIPA solutions with $23 \mathrm{~mm}$ DTAC also decreases steeply at $\sim 37^{\circ} \mathrm{C}$, in contrast to the case for $I_{1} / I_{3}$ for the same solution. It is more important to note that the values of $I_{1} / I_{3}$ or the $\lambda_{\max }$ for the two PNIPA samples, M5 and R5, are in good agreement with each other at either value of [DTAC]. This agreement between the two PNIPA samples indicates that the difference in the chain-end groups of PNIPA does not cause the difference in the microenvironmental polarity surrounding the probe molecules if DTAC (instead of SDS) is added to the solutions. Considering the fact that the sample R5 has an anionic sulfonate group at its chain end, the cationic DTAC molecules are expected to be adsorbed selectively onto the negatively charged chain ends of the sample R5, and then the hydrophobic alkyl chains of DTAC around the chain end diminish its hydrophilicity, whereas this phenomenon does not occur for the sample M5 having a nonionic hydrophobic chain end. Consequently, the difference in the hydrophobicity of the chain end between the two PNIPA samples, M5 and R5, may be considered to virtually disappear in the solutions containing DTAC. As the result, the values of $I_{1} / I_{3}$ or the $\lambda_{\max }$ for the solutions of the two samples agree well with each other, in the presence of DTAC, as shown in Figure 7.

Finally, we note that the decrease in the cloud point for the aqueous solutions of the $\mathrm{R}$ sample containing DTAC from its value for the surfactant-free solution, as seen in Figure 1 for lower $c_{\mathrm{s}}(\lesssim 10 \mathrm{~mm})$, may be considered to arise for the same reason as above, that is, the DTAC molecules selectively adsorbed onto the negatively charged chain end of the $\mathrm{R}$ sample may diminish the hydrophilicity of its chain end.

\section{CONCLUSION}

We determined the $I_{1} / I_{3}$ of pyrene and the $\lambda_{\max }$ of 1-pyrenecarboxaldehyde for aqueous solutions of two PNIPA samples, M5 having a hydrophobic chain-end group and R5 having a negatively charged hydrophilic chain-end group, with the addition of two types of surfactants, anionic SDS or cationic DTAC. For all of the solutions, the $I_{1} / I_{3}$ and the $\lambda_{\max }$ that were determined at $25.0^{\circ} \mathrm{C}$ decreased abruptly at the $\mathrm{CAC}$ at which the surfactant molecules begin to bind to the PNIPA chains to form the PNIPA-surfactant complex micelles. The CAC for SDS in PNIPA solutions was found to be much lower than that for DTAC. The difference in the CACs between SDS and DTAC may possibly cause the difference in the value of the $c_{\mathrm{s}}$ at which the cloud point in the aqueous PNIPA solutions with surfactant begins to increase from its value for the surfactant-free solutions.

For the aqueous solutions of the two PNIPA samples with 0.35 and $3.5 \mathrm{~mm}$ SDS, and with 11 and $23 \mathrm{~mm}$ DTAC, the $I_{1} / I_{3}$ and the $\lambda_{\max }$ were determined over a range of temperatures from $25.0^{\circ} \mathrm{C}$ to above the cloud points in the respective solutions. Because the CACs for SDS and DTAC in aqueous PNIPA solutions were found to be $\sim 0.9 \mathrm{~mm}$ and $\sim 15 \mathrm{~mm}$, respectively, it is possible that PNIPA-surfactant complex micelles are formed with $3.5 \mathrm{~mm}$ SDS and $23 \mathrm{~mm}$ DTAC, but not with $0.35 \mathrm{~mm}$ SDS and $11 \mathrm{~mm}$ DTAC. In addition, the $I_{1} / I_{3}$ and the $\lambda_{\max }$ for the solutions containing SDS are lower for the sample M5 than for the sample R5, with either 0.35 or $3.5 \mathrm{~mm}$ SDS. This indicates that chain-end groups of PNIPA may affect the microenvironmental polarity surrounding the fluorescence-probe molecules solubilized in the solutions containing SDS. On the other hand, the values of the $I_{1} / I_{3}$ and the $\lambda_{\max }$ for the solutions of the two PNIPA samples, M5 and R5, containing DTAC agree well with each other, for both 11 and $23 \mathrm{~mm}$ DTAC. The agreement of the value of $I_{1} / I_{3}$ and the $\lambda_{\max }$ in the solutions containing DTAC between the two PNIPA samples may be due to the cationic DTAC molecules being adsorbed selectively onto the negatively charged chain ends of the sample R5. 
The hydrophobic alkyl chains of DTAC around the chain end diminish its hydrophilicity, so that the difference in the hydrophobicity of the chain end between the two PNIPA samples virtually disappears in solutions containing DTAC.

\section{CONFLICT OF INTEREST}

The authors declare no conflict of interest.

1 Kawaguchi, T., Kojima, Y., Osa, M. \& Yoshizaki, T. Cloud points in aqueous poly(N-isopropylacrylamide) solutions. Polym. J. 40, 455-459 (2008).

2 Kawaguchi, T., Kojima, Y., Osa, M. \& Yoshizaki, T. Primary structure of poly( $N$-isopropylacrylamide) synthesized by radical polymerization. Effects of polymerization solvents. Polym. J. 40, 528-533 (2008).

3 Kobayashi, K., Yamada, S., Nagaoka, K., Kawaguchi, T., Osa, M. \& Yoshizaki, T. Characterization of linear poly( $\mathrm{N}$-isopropylacrylamide) and cloud points in its aqueous solutions. Polym. J. 41, 416-424 (2009).

4 Kawaguchi, T., Kobayashi, K., Osa, M. \& Yoshizaki, T. Is a 'cloud-point curve' in aqueous poly( $\mathrm{N}$-isopropylacrylamide) solution binodal? J. Phys. Chem. B 113, 5440-5447 (2009).

5 Ise, T., Nagaoka, K., Osa, M. \& Yoshizaki, T. Cloud points in aqueous solutions of poly( $N$-isopropylacrylamide) synthesized by aqueous redox polymerization. Polym. J. 43, 164-170 (2011).

6 Schild H. G. Poly( $N$-isopropylacrylamide): experiment, theory and application. Prog. Polym. Sci. 17: 163-249 (1992).

7 Schild, H. G. \& Tirrell, D. A. Sodium 2-(N-dodecylamino)naphthalene-6-sulfonate as a probe of polymer-surfactant interaction. Langmuir 6, 1676-1679 (1990).

8 Schild, H. G. \& Tirrell, D. A. Interaction of poly( $N$-isopropylacrylamide) with sodium $n$-alkyl sulfates in aqueous solution. Langmuir 7, 665-671 (1991).
9 Meewes, M., Rička, J., de Silva, M., Nyffenegger, R. \& Binkert, Th. Coil-globule transition of poly( $\mathrm{N}$-isopropylacrylamide). A study of surfactant effects by light scattering. Macromolecules 24, 5811-5816 (1991).

10 Walter, R., Rička, J., Quellet, Ch., Nyffenegger, R. \& Binkert, Th. Coil-globule transition of poly( $N$-isopropylacrylamide). A study of polymer-surfactant association. Macromolecules 29, 4019-4028 (1996).

11 Kokufuta, E., Zhang, Y., Tanaka, T. \& Mamada, A. Effects of surfactants on the phase transition of poly(N-isopropylacrylamide) gel. Macromolecules 26, 1053-1059 (1993).

$12 \mathrm{Wu}, \mathrm{C}$., Zhou, S. Effects of surfactants on the phase transition of poly( $\mathrm{N}$-isopropylacrylamide) in water. J. Polym. Sci. B 34, 1597-1604 (1996).

13 Abuin, E., Leon, A., Lissi, E. \& Varas, J. M. Binding of sodium dodecylsulfate to poly( $N$-isopropylacrylamide) microgels at different temperatures. Colloids Surf. A 147, 55-65 (1999).

14 Al-Manasir, N., Zhu, K., Kjøniksen, A., Knudsen, K. D. \& Nyström, B. Effects of addition of anionic and cationic surfactants to poly( $N$-isopropylacrylamide) microgels with and without acrylic acid groups. Colloid Polym. Sci. 290, 931-940 (2012).

15 Uehara, N. \& Ogawa, M. Interaction of poly( $N$-isopropylacrylamide) with sodium dodecyl sulfate below the critical aggregation concentration. Langmuir 2014; 30: 6367-6372.

16 Osa, M., Shiraki, H., Morinaga, U. \& Yoshizaki, T. Effects of surfactants on cloud points in aqueous poly( $\mathrm{N}$-isopropylacrylamide) solutions. Polym. J. 45, 681-684 (2013).

17 Wooten, W. C., Blanton, R. B. \& Coover, H. W. Effect of pH on homopolymerization of $\mathrm{N}$-isopropylacrylamide. J. Polym. Sci. 25, 403-412 (1957).

18 Wu, W., Wang, W., Li, S., Wang, J., Zhang, Q., Li, X., Luo, X. \& Li, J. Physiological $\mathrm{pH}$-triggered morphological transition of amphiphilic block copolymer self-assembly. J. Polym. Res. 21, 494 (2014).

19 Goddard, E. D. Polymer-surfactant interaction part I. Uncharged water-soluble polymers and charged surfactants. Colloids Surf. 19, 255-300 (1986).

20 Ringsdorf, H., Venzmer, J. \& Winnik, F. M. Fluorescence studies of hydrophobically modified poly( $N$-isopropylacrylamides). Macromolecules 24, 1678-1686 (1991).

21 Kalyanasundaram, K. \& Thomas, J. K. Solvent-dependent fluorescence of pyrene-3carboxaldehyde and its applications in the estimation of polarity at micelle-water interfaces. J. Phys. Chem. 81, 2176-2180 (1977).

22 Holmberg K., Jönsson B., Kronberg B. \& Lindman B. Surfactants and Polymers in Aqueous Solution, 2nd edn (John Wiley \& Sons Ltd: Chichester, England, 2003). 